\title{
Prediction of texture of raw poultry meat by visible and near-infrared reflectance
} spetroscopy

\begin{abstract}
Near-infrared (NIR) spectroscopy is a non-destructive, low cost and fast measurement technique that is required to improve the meat texture quality prediction. In this research, visible/NIR spectroscopy has been used for the prediction of raw chicken meat texture from different types of chickens by referring to the reference data obtained from destructive measurement using a Volodkevich Bite Jaws texture analyser. The Partial Least Squares analysis shows that the prediction accuracy is higher for the Az-Zain village organic chickens (85-95\%) than for village chickens (42-68\%) and broiler chickens (42-44\%). The high prediction accuracy and low absorbance spectra of Az-Zain village organic chickens compared to broiler and village chickens could be correlated with the food composition of the chicken meal.
\end{abstract}

Keyword: Chicken meat; Tenderness; NIRS; Volodkevich Bite Jaws; Partial least squares 